Print- ISSN: 1391-586X, E- ISSN: 2602-9030, Copyright (C) 2018 by Faculty of Science, Eastern University, Sri Lanka.

\title{
A MATHEMATICAL AND NUMERICAL ANALYSIS OF A COMPARTMENTAL MODEL FOR A SINGLE STRAIN DENGUE EPIDEMIC IN THE PRESENCE OF VACCINATION
}

\author{
Madhusankha, B. H. I. ${ }^{*}$, Ariyawansa, T. S. V. ${ }^{1}$, and Perera, S. S. N. ${ }^{1}$ \\ ${ }^{1}$ Research \& Development Centre for Mathematical Modelling, Faculty of Science, University \\ of Colombo, Sri Lanka
}

\begin{abstract}
Dengue disease has presently emerged as an alarming public health concern of mounting significance with the unprecedented number of cases reported worldwide annually, especially from the tropics and subtropics. It is currently considered as the most critical vector borne viral disease in the world due to the acute health, social and economic issues that it causes in the endemic countries. Further, human mobility, climate change, rapid urbanization and public unawareness have led to a substantial dissemination of dengue in new geographical settings with explosive outbreaks. Also, the disease burden has aggravated due to the unavailability of specific clinical therapeutics and effective vaccines for the dengue disease. In such a context, this paper intends to investigate the dynamics of dengue disease in the presence of hypothetical vaccination strategies. Thus, we use a classical compartmental model with mathematical and numerical analyses in order to emphasize the importance of vaccination as an effective measure of disease control. We are hence of the belief that this paper will be a source of motivation for the medical scientists involved in the development of vaccine candidates for the dengue disease.
\end{abstract}

Keywords: dengue disease dynamics; hypothetical vaccination; single strain SIR models; equilibrium solutions; basic reproduction number.

${ }^{*}$ Corresponding author: bhindunil.sl@gmail.com https://orcid.org/0000-0002-2769-8299 


\section{INTRODUCTION}

In accordance with the World Health Organization (WHO), a study on the prevalence of dengue estimates that 3.9 billion people in 128 countries around the world are currently at risk for infection with the dengue virus [13]. Dengue disease has grown dramatically in the recent past thus posing a devastating threat on the socio-economic life of most of the countries in the tropical and sub-tropical regions of the world. It is an infectious disease transmitted to humans mainly through the bites of female Aedes aegypti mosquitos. Dengue is caused by four different serotypes of the DENV virus group, namely, DENV 1, DENV 2, DENV 3 and DENV 4 which are serologically and genetically related $[12,13]$. An infection from one serotype gives life-long immunity against that serotype, but an individual may get sequentially infected with thethree remaining serotypes. There are three forms of the dengue disease as Dengue Fever (DF) which is milder, and the more severe forms of Dengue Hemorrhagic Fever (DHF) and Dengue Shock Syndrome (DSS) which reflect a high level of disease severity requiring intensive medical care $[12,13]$.

Due to the unavailability of specific clinical treatments for dengue, vector control measures are currently regarded as the mostly used measure of disease prevention [13, 14]. The medical scientists have thus been undertaking extensive research into the development of a tetravalent vaccine which has the ability to provide life-long immunity against all the four serotypes simultaneously [14]. At a time when the attention of medical researchers is widely attracted towards the experimentation of dengue vaccines, it appears to be of timely importance to explore the impact of vaccination on the dynamics of dengue disease. Therefore, the objective of this study is to analyze the changing dynamics of dengue disease in the presence of hypothetical vaccination strategies. So, we use a compartmental model in order to examine the dynamics of a single strain dengue epidemic under the effect of hypothetical vaccination.

The classical compartmental model (referred to as the SIR model) is generally composed of three basic compartments, namely, the Susceptible $(S)$, Infected $(I)$ and Recovered/Removed $(R)$ which represents a classification of the population according to the health status (with respect to the disease) of the individuals at time t. This model has been successfully used in the literature to examine single strain disease dynamics as evident in such works as Derouich et al. [5], Esteva and Vargas [6], Yaacob [15], Side and Noorani [9], etc., while the use of multi-strain epidemiological models appears in Augiar et al. [1], Augiar et al. [2], Nuraini et al. [7], etc. The importance of vaccination 
has previously been taken into consideration in many works including Deroiuch et al. [5], Augiar et al. [1] and Augiar et al. [2]. Epidemiologists, however, consolidate that the single strain model is insufficient for a closer scrutiny of the complex and varying dynamics of dengue disease, and have thus stressed the need for a minimalistic multi strain dengue model, especially in explaining the complications governing secondary dengue infections.

In our study, we conduct both mathematical and numerical investigations so as to explore the impact of vaccination coverage and vaccine efficacy on the dynamics of potential dengue epidemics. Strikingly, the study contributed to certain significant findings on the importance of vaccination in the control of dengue disease. It is further expected that this study will be of great interest to those engaged in the process of developing vaccines against dengue. A detailed explanation of the model is presented in Section 2. The mathematical analysis in Section 3 probes into some important results on the basic reproduction number and the equilibria of the system of equations pertaining to the present model. Moreover, the model simulations appearing in Section 4 focuses on a numerical study on the dynamics of dengue using the distributions of infectives and phase plane diagrams subject to the variations of the chosen parameters. Finally, the conclusion in Section 5 sums up the important results derived from the exploration, and also provides certain suggestions for future research.

\section{MODEL DEVELOPMENT}

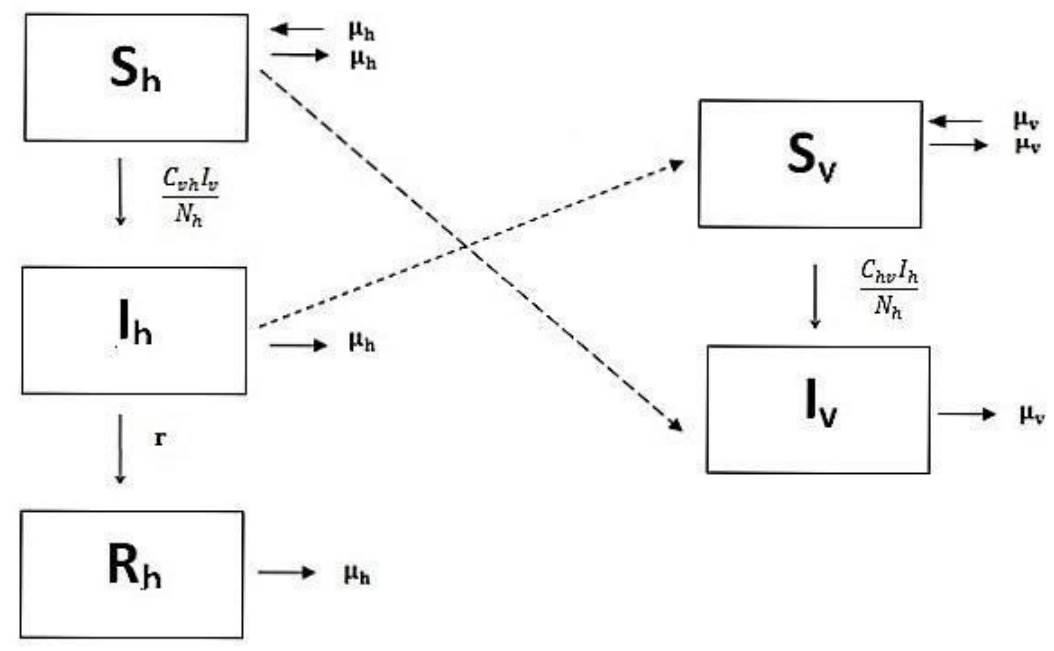

Figure 1: The schematic diagram for the single strain host-vector dengue model. 
Figure 1 displays the classification of the human population into three mutually exclusive compartments namely the Susceptible $\left(S_{h}\right)$, Infected $\left(I_{h}\right)$ and Recovered $\left(R_{h}\right)$, and that of the vector population into two mutually exclusive classes: Susceptible $\left(S_{v}\right)$ and Infected $\left(I_{v}\right)$. A recovered compartment for the vectors is not considered here due to the very short life span of the mosquitoes. The rates of transfer between the compartments are mathematically represented as derivatives of the sizes of the compartments with respect to time, which yield systems of ordinary differential equations. It is assumed that the size of the population is a large number, and remains constant (i.e. birth rate $=$ mortality rate) over the duration of the entire epidemic. Two other important assumptions of the model are that the population is homogeneous, and that the contacts between host and vector populations are also homogeneous $[3,8]$.

The parameters $\mu_{\mathrm{h}}$ and $\mu_{\mathrm{v}}$ denote the demography rates for the hosts and vectors respectively. The recovery rate for humans is denoted by $r$. Further, $C_{v h}$ and $C_{h v}$ are the effective contact rates from vectors to humans and humans to vectors respectively, and are given explicitly by the equations, $C_{v h}=P_{v h} b$ and $C_{h v}=P_{h v} b$ respectively. Here, $P_{v h}$ and $P_{h v}$ are the transmission probabilities from vectors to humans and humans to vectors respectively, and $b$ is the biting rate. In the present model which follows the structure used by Derouich et al. [5], we introduce a vaccine efficacy factor $(\theta)$ and a vaccination rate $(v)$, as in the two strain dengue model used by Augiar et al. [2]. The vaccination coverage $(p)$ represents the proportion of the population that is vaccinated whereas the vaccine efficacy factor $(\theta)$ parameterizes the effectiveness of the vaccine as a percentage of the level of immunity that it provides with a vaccinated individual. Further, we assume that the vaccine is implemented with vaccination rate $v$ so that the vaccination period is $\frac{1}{v}$. Also, the dynamics of human-vector interaction are presented in the system (1). 


$$
\begin{aligned}
& \frac{d S_{h}}{d t}=\mu_{h} N_{h}-\left(\mu_{h}+\theta P v+\frac{C_{p h} I_{v}}{N_{h}}\right) s_{h} \\
& \frac{d I_{h}}{d t}=\left(\frac{C_{v h} I_{v}}{N_{h}}\right) s_{h}-\left(\mu_{h}+r\right) I_{h} \\
& \frac{d R_{h}}{d t}=\theta P v S_{h}+r I_{h}-\mu_{h} R_{h} \\
& \frac{d s_{v}}{d t}=\mu_{v} N_{v}-\frac{\left(\mu_{v} N_{h}+C_{h} J_{h}\right)}{N_{h}} S_{v} \\
& \frac{d I_{v}}{d t}=\frac{\left(C_{h v} I_{h}\right)}{N_{h}} S_{v}-\mu_{v} I_{v}
\end{aligned}
$$

with $s_{h}+I_{h}+R_{h}=N_{h}$ and $s_{v}+I_{v}=N_{v}$.

Now we introduce dimensionless quantities and reduce (1) to a system of three equations.

$$
\begin{aligned}
& \frac{d S_{h}}{d t}=\mu_{h}-\left(\mu_{h}+\theta P v+n C_{v h} I_{v}\right) S_{h} \\
& \frac{d I_{h}}{d t}=n C_{v h} I_{v} S_{h}-\left(\mu_{h}+r\right) I_{h} \\
& \frac{d I_{v}}{d t}=C_{h v} I_{h}\left(1-I_{v}\right)-\mu_{v} I_{v} \text { where } n=\frac{N_{v}}{N_{h}} \text { and } 0 \leq S_{h}, I_{h}, I_{v} \leq 1,
\end{aligned}
$$

with initial conditions $S_{h}(0) \geq 0, I_{h}(0) \geq 0$ and $I_{v}(0) \geq 0$.

\section{MATHEMATICAL ANALYSIS OF THE MODEL}

\subsection{Basic Reproduction Number $\left(R_{0}\right)$}

In epidemiology, the basic reproduction number, denoted by $R_{0}$, is defined as the expected number of secondary infections generated by a single case in a completely susceptible population during its entire infectious lifetime [10, 11]. So, the basic reproduction number provides an idea regarding the initial spread of a disease in a susceptible population in the presence of a single infectious individual. The higher the value of $R_{0}$, the more transmissible the disease, and is hence more difficult to control the epidemic [11].

\subsection{Computation of the Basic Reproduction Number $\left(R_{0}\right)$}

Now we calculate the basic reproduction number $\left(R_{0}\right)$, which we use subsequently in order to derive a condition for the stability of the equilibrium solutions of the system of 
equations in (2). The popular method known as the next generation matrix is used here in order to compute $R_{0}$.

Since only the $I_{h}$ and $I_{V}$ compartments contribute to the propagation of the disease, we consider only those two compartments in composing the next generation matrix. Observe that

Gain $I_{h}=n C_{v h} I_{v} S_{h}$, Loss $I_{h}=\left(\mu_{h}+r\right) I_{h}$, Gain $I_{v}=C_{h v} I_{h}$ and Loss $I_{v}=C_{h v} I_{h} I_{v}+\mu_{v} I_{v}$.

Then the Infection Matrix is given by $F=\left(\begin{array}{cc}\frac{\partial I_{h} G a i n}{\partial I_{h}} & \frac{\partial I_{h} G a i n}{\partial I_{v}} \\ \frac{\partial I_{v} G a i n}{\partial I_{h}} & \frac{\partial I_{v} G a i n}{\partial I_{v}}\end{array}\right)=\left(\begin{array}{cc}0 & C_{h v} \\ n C_{v h} S_{h} & 0\end{array}\right)$

and the Transition Matrix is $V=\left(\begin{array}{cc}\frac{\partial I_{h} L o s s}{\partial I_{h}} & \frac{\partial I_{h} L o s s}{\partial I_{v}} \\ \frac{\partial I_{v} L o s s}{\partial I_{h}} & \frac{\partial I_{v} L o s s}{\partial I_{v}}\end{array}\right)=\left(\begin{array}{cc}\mu_{h}+r & C_{h v} I_{v} \\ 0 & C_{h v} I_{h}+\mu_{v}\end{array}\right)$.

The Next Generation Matrix is obtained as follows,

$$
G=F V^{-1}=\left(\begin{array}{cc}
0 & C_{h v} \\
n C_{v h} S_{h} & 0
\end{array}\right)\left(\begin{array}{cc}
\frac{1}{\mu_{h}+r} & \frac{-C_{h v} I_{v}}{\left(\mu_{h}+r\right)\left(C_{h v} I_{h}+\mu_{v}\right)} \\
0 & \frac{1}{C_{h v} I_{h}+\mu_{v}}
\end{array}\right) \text {. }
$$

Substituting the virus free equilibrium $E_{o}=\left(\frac{\mu_{h}}{\mu_{h}+\theta P v^{\prime}}, 0,0\right)$ (which we will derive in Section 3.4) yields;

$G=F V^{-1}=\left(\begin{array}{cc}0 & C_{h v} \\ n C_{v h} S_{h} & 0\end{array}\right)\left(\begin{array}{cc}\frac{1}{\mu_{h}+r} & 0 \\ 0 & \frac{1}{\mu_{v}}\end{array}\right)=\left(\begin{array}{cc}0 & \frac{C_{h v}}{\mu_{v}} \\ \frac{n C_{v h} \mu_{h}}{\left(\mu_{h}+r\right)\left(\mu_{h}+\theta P v\right)} & 0\end{array}\right)$.

Since $R_{\sigma}$ is defined as the spectral radius of $G$, we consider the characteristic equation of $G$ given by,

$|G-\lambda|=\left|\lambda^{2}-\frac{n \mu_{h} C_{v h} C_{h v}}{\mu_{v}\left(\mu_{h}+r\right)\left(\mu_{h}+\theta p_{v j}\right]}\right|=0$.

Hence we have $\lambda= \pm \sqrt{\frac{n \mu_{h} C_{v h} C_{h v}}{\mu_{v}\left(\mu_{h}+r\right)\left(\mu_{h}+\theta p v\right)}}$.

Therefore, $R_{o}=r(G)=\sqrt{\frac{n \mu_{h} C_{v h} C_{h v}}{\mu_{v}\left(\mu_{h}+r\right)\left(\mu_{h}+\theta P v\right)}}$. 


\section{3 Sensitivity of the Basic Reproduction Number with respect to the Vaccination Parameters}

The basic reproduction number is considered as an important tool in the mathematical analyses of infectious diseases as this statistic provides significant insight into disease dynamics. The value of $R_{0}$ depends on the parameters used in the model. The implications of the dependence of $R_{0}$ on such parameters are pivotal in understanding disease spread and planning intervention strategies. So, we present the following important proposition on the relationship between the basic reproduction number and the three vaccination parameters in the model.

Proposition 1: $R_{o}$ is a decreasing function of the vaccination coverage $(\mathrm{P})$, vaccine efficacy $(\theta)$ and vaccination rate $(v)$.

Remark 1: Note that the partial derivatives $\frac{\partial R_{0}}{\partial P}=-\left(\frac{1}{2}\right) \theta v\left(\mu_{h}+\theta P v\right)^{-3 / 2} \sqrt{\frac{n \mu_{h} C_{v h} C_{h v}}{\mu_{v}\left(\mu_{h}+r\right)}}<0$,

$\frac{\partial R_{0}}{\partial \theta}=-\left(\frac{1}{2}\right) P V\left(\mu_{h}+\theta P v\right)^{-3 / 2} \sqrt{\frac{n \mu_{h} C_{v h} C_{h v}}{\mu_{v}\left(\mu_{h}+r\right)}}<0$ and

$\frac{\partial R_{0}}{\partial V}=-\left(\frac{1}{2}\right) P \theta\left(\mu_{h}+\theta P v\right)^{-3 / 2} \sqrt{\frac{n \mu_{h} C_{v h} C_{h v}}{\mu_{v}\left(\mu_{h}+r\right)}}<0$.

Proposition 1 indicates that the dengue disease can greatly be controlled by taking such measures as widening the cohort of the population that is vaccinated, enhancing the efficacy of the vaccines administered, and increasing the vaccination rate. The implication here is that vaccination is an effective measure of controlling the spread of dengue.

\subsection{The Existence and Stability Analysis of Equilibria in the Model}

In the present segment, we examine the equilibrium solutions of the system of ODE's in the model, and attempt to interpret them in relation to the nature of the epidemic. So, we have the following important propositions.

Proposition 2: Let $A=\left\{\left(S_{h}, I_{h}, I_{v}\right) ; 0 \leq I_{v} \leq 1,0 \leq I_{h}, 0 \leq S_{h},\left(\frac{\mu_{h}+\theta P_{v}}{\mu_{h}}\right) S_{h}+I_{h} \leq 1\right\}$. Then the system (2) comprises of two equilibrium solutions namely the disease free equilibrium $E_{o}=\left(\frac{\mu_{h}}{\mu_{h}+\theta p_{v}}, 0,0\right)$ and the endemic equilibrium $E_{1}=$ 
$\left(\frac{x}{y}, \frac{1}{\mu_{h}+r} \frac{z}{y}, \frac{1}{n C_{v h}} \frac{z}{x}\right)$ where $\mathrm{X}=\mu_{h}+\left(\mu_{h}+r\right) t, \quad \mathrm{Y}=\mu_{h}+\theta P v+n C_{v h}, \quad \mathrm{Z}$ $=n C_{v h} \mu_{h}-\left(\mu_{h}+r\right)\left(\mu_{h}+\theta P V\right) t$ and $n=\frac{N_{v}}{N_{h}}, \mathrm{t}=\frac{\mu_{v}}{C_{h v}}$.

Proof: Consider the system (2). For equilibrium points we have,

$\mu_{h}-\left(\mu_{h}+\theta P v+n C_{v h} I_{v}\right) S_{h}=0$

$n C_{v h} I_{v} S_{h}-\left(\mu_{h}+r\right) I_{h}=0$

$C_{h v} I_{h}\left(1-I_{v}\right)-\mu_{v} I_{v}=0$

By simplifying, we have $E_{o}=\left(\frac{\mu_{h}}{\mu_{h}+\theta p v}, 0,0\right)$, and $E_{1}=\left(\frac{x}{y}, \frac{1}{\mu_{h}+r} \frac{z}{y}, \frac{1}{n c_{v h}} \frac{z}{x}\right)$

where $\mathrm{X}=\mu_{h}+\left(\mu_{h}+r\right) t, \mathrm{Y}=\mu_{h}+\theta P v+n C_{v h}$ and $\mathrm{Z}=n C_{v h} \mu_{h}-\left(\mu_{h}+r\right)\left(\mu_{h}+\theta P v\right) t$

Remark 2: The disease free equilibrium $E_{o}$ relates to the disease free state where the disease dies out leaving the population free from the infection while the endemic equilibrium $E_{1}$ corresponds to the endemic state where the disease takes hold in the community.

Proposition 3: The endemic equilibrium disappears for $R_{o} \leq 1$, thus leaving the disease free equilibrium the only equilibrium point of the system. For $R_{o}>1$, the endemic equilibrium also becomes an equilibrium solution of the system.

Proof: Observe that

$Z=n C_{w h} \mu_{h}-\left(\mu_{h}+r\right)\left(\mu_{h}+\theta P v\right) \frac{\mu_{v}}{C_{h v}}=n C_{v h} \mu_{h}\left[1-\frac{\mu_{v}\left(\mu_{h}+r\right)\left(\mu_{h}+\theta P V\right)}{n \mu_{h} C_{v h} C_{h v}}\right]=$ $n C_{w h} \mu_{h}\left(1-\frac{1}{R_{0}^{2}}\right)>0$

only if $R_{o}>1$.

Thus $E_{1}$ exists only for $R_{o}>1$.

Remark 3: Proposition 3 suggests that the disease will fade away if $R_{o} \leq 1$, and that the disease will persist (i.e. there will be an epidemic) if $R_{0}>1$.

Proposition 4: $E_{o}$ is locally asymptotically stable for $R_{o} \leq 1$. 
Proof: The Jacobian matrix for $E_{0}$ reads as,

$$
J\left(E_{0}\right)=\left[\begin{array}{ccc}
-\left(\mu_{h}+\theta P v\right) & 0 & -n C_{w h} \frac{\mu_{h}}{\mu_{h}+\theta P v} \\
0 & -\left(\mu_{h}+r\right) & n C_{v h} \frac{\mu_{h}}{\mu_{h}+\theta P v} \\
0 & C_{h v} & -\mu_{v}
\end{array}\right]
$$

The characteristic equation of $J\left(E_{0}\right)$ is $a_{0} \lambda^{3}+a_{1} \lambda^{2}+a_{2} \lambda+a_{3}=0$

where $a_{0}=1, a_{1}=\mu_{v}+2 \mu_{h}+r+\theta P v, a_{2}=$

$\left(\mu_{h}+\theta P v\right)\left(\mu_{v}+\mu_{h}+r\right)+\left[\mu_{v}\left(\mu_{h}+r\right)-n C_{h v} C_{v h} \frac{\mu_{h}}{\mu_{h}+\theta P v}\right]$ and $a_{3}=$

$\left(\mu_{h}+\theta P v\right)\left[\mu_{v}\left(\mu_{h}+r\right)-n C_{h v} C_{v h} \frac{\mu_{h}}{\mu_{h}+\theta P v}\right]$.

Then the eigenvalues of $J\left(E_{o}\right)$ are given by,

$\lambda_{1}=-\left(\mu_{h}+\theta P v\right)$

$\lambda_{2}=\frac{-\left(\mu_{v}+\mu_{h}+r\right)+\sqrt{\left(\mu_{v}+\mu_{h}+r\right)^{2}-4\left[\mu_{v}\left(\mu_{h}+r\right)-n C_{h v} C_{v h} \frac{\mu_{h}}{\mu_{h}+\theta P v}\right]}}{2}$

$\lambda_{3}=\frac{-\left(\mu_{v}+\mu_{h}+r\right)-\sqrt{\left(\mu_{v}+\mu_{h}+r\right)^{2}-4\left[\mu_{v}\left(\mu_{h}+r\right)-n C_{h v} C_{v h} \frac{\mu_{h}}{\mu_{h}+\theta P_{v}}\right]}}{2}$.

$E_{o}$ is locally asymptotically stable if all three eigenvalues have negative real parts. Notice that $\lambda_{1}$ is a real number and $\lambda_{1}<0$. Further, it is easy to verify that $\lambda_{2}<0$ and $\lambda_{3}<0$ for $R_{o} \leq 1$.

Thus the proposition.

Proposition 5: $E_{o}$ is globally asymptotically stable for $R_{o} \leq 1$.

Proof: In order to establish the global stability of $E_{o}$, we construct a Liyapunov function by appropriately modifying the Liyapunov function given by Derouich et al. [5].

Consider the continuously differentiable function $V: A \rightarrow \mathbb{R}$ given by

$V=\frac{c_{v h}}{\mu_{v}} I_{v}+\left(\frac{\mu_{h}+\theta P v}{\mu_{h}}\right) I_{h}$. 
Observe that $V\left(E_{0}\right)=0$ and $V(x)>0$ for $x \in A-\left\{E_{0}\right\}$. Thus, $V$ is positive definite.

Now we need to show that $\frac{d \mathrm{~V}(\mathrm{x})}{d t}<0$ for $x \in A-\left\{E_{0}\right\}$.

Let $x \in A-\left\{E_{0}\right\}$. Then we have

$$
\begin{aligned}
\frac{d V(x)}{d t} & =n \frac{C_{v h}}{\mu_{v}} \frac{d I_{v}}{d t}+\left(\frac{\mu_{h}+\theta P v}{\mu_{h}}\right) \frac{d I_{h}}{d t} \\
& =\frac{C_{v h}}{\mu_{v}}\left(C_{h v} I_{h}\left(1-I_{v}\right)-\mu_{v} I_{v}\right)+\left(\frac{\mu_{h}+\theta P v}{\mu_{h}}\right)\left(n C_{v h} I_{v} S_{h}-\left(\mu_{h}+r\right) I_{h}\right) \\
& =-\left\{n C_{v h}\left(1-\left(\frac{\mu_{h}+\theta P v}{\mu_{h}}\right) S_{h}\right) I_{v}+\frac{n C_{h v} C_{v h}}{\mu_{v}}\left(\frac{\mu_{v}\left(\mu_{h}+r\right)\left(\mu_{h}+\theta P v\right)}{n \mu_{h} C_{h v} C_{v h}}-1+I_{h}\right)\right\} .
\end{aligned}
$$

It is easy to observe that $1-\left(\frac{\mu_{h}+\theta P v}{\mu_{h}}\right) S_{h}>0$ by the definition of the set A.

Further, $R_{o} \leq 1$ yields $\frac{\mu_{v}\left(\mu_{h}+r\right)\left(\mu_{h}+\theta P v\right)}{n \mu_{h} C_{h v} c_{p h}}-1=\frac{1}{R_{0}^{2}}-1>0$.

$\therefore \frac{d V(x)}{d t}<0$.

Thus the existence of the Liyapunov function concludes the proof.

Proposition 6: $E_{1}$ is locally asymptotically stable for $R_{0}>1$.

Proof: The Jacobian matrix for $E_{1}$ is,

$$
J\left(E_{1}\right)=\left[\begin{array}{ccc}
-\left(\mu_{h}+\theta P v+\frac{Z}{X}\right) & 0 & -n C_{v h} \frac{X}{Y} \\
\frac{Z}{X} & -\left(\mu_{h}+r\right) & n C_{v h} \frac{X}{Y} \\
0 & C_{h v}\left(1-\frac{1}{n C_{v h}} \frac{Z}{X}\right) & -\left(\mu_{v}+\frac{C_{h v}}{\left(\mu_{h}+r\right)}\right) \frac{Z}{Y}
\end{array}\right]
$$

The characteristic equation of $J\left(E_{1}\right)$ is $a_{0} \lambda^{3}+a_{1} \lambda^{2}+a_{2} \lambda+a_{3}=0$ where

$$
\begin{aligned}
& \left.a_{0}=1, a_{1}=2 \mu_{h}+\mu_{v}+r+\theta P v+\frac{Z}{X}+\frac{C_{h v}}{\left(\mu_{h}+r\right)}\right) \frac{Z}{Y}, \\
& \left.a_{2}=C_{h v} \frac{Z}{Y}+\left(\mu_{h}+\theta P v+\frac{Z}{X}\right)\left(\mu_{h}+\mu_{v}+r+\frac{C_{h v}}{\left(\mu_{h}+r\right)}\right) \frac{Z}{Y}\right) \text { and } a_{3}=C_{h v} Z .
\end{aligned}
$$

Local stability of the point is satisfied if all eigenvalues have negative real roots.

According to Routh-Hurwitz criterion, all roots of $\mathrm{P}(\lambda)$ have negative real parts if $a_{1}>0, a_{2}>0, a_{3}>0$ and $a_{1} a_{2}>a_{3}$. 
Since $\mathrm{Z}>0$ for $R_{o}>1$, it is easy to observe that $a_{1}>0, a_{2}>0, a_{3}>0$.

Further it can be shown that

$$
\begin{aligned}
& a_{1} a_{2}=\left(2 \mu_{h}+\mu_{v}+r+\theta P v+\frac{Z}{X}+\frac{C_{h v}}{\left(\mu_{h}+r\right)} \frac{Z}{Y}\right)\left[C_{h v} \frac{Z}{Y}+\left(\mu_{h}+\theta P v+\frac{Z}{X}\right)\left(\mu_{h}+\mu_{v}+r+\right.\right. \\
& \left.\left.\frac{C_{h v}}{\left(\mu_{h}+r\right)} \frac{Z}{Y}\right)\right] \\
& =C_{h v} Z+k>C_{h v} Z=a_{3} \text { where } k>0 .
\end{aligned}
$$

Thus $E_{l}$ is locally asymptotically stable for $R_{o}>1$.

\section{NUMERICAL RESULTS AND DISCUSSION}

In order to numerically solve the systems of ODE's in the model, we use the ode45 function which is the standard solver of MATLAB for ordinary differential equations. The ode45 function is based on a Runge-Kutta method which uses a variable time step. For the analysis, we vary the parameters $p$ and $\theta$ in the model, and then observe the consequent effect on the spread, prevalence and magnitude of potential epidemic outbreaks. First, we observe the changing behavior of the curves of infected humans $\left(I_{h}\right)$ and infected vectors $\left(I_{v}\right)$ subject to the variation of vaccination coverage $(\mathrm{p})$ and vaccine efficacy $(\theta)$. Then, we use the rand () function in MATLAB in order to obtain the mean and standard deviation of the distribution of infectives in both human and vector populations over a period of one year. The rand() function on MATLAB was used to randomly vary the parameters $p$ and $\theta$ in the specified range. Finally, we study the behavior of two important phase portrait simulations. The initial conditions for the three variables $\left[S_{h}, I_{h}, I_{v}\right]$ are given by the vector $[0.9999,0.0001,0]$. A description of the parameters and their values used in the present simulations are given in Table 1. 
Table 1: The set of parameters for the single strain epidemiological model with descriptions, values and references.

\begin{tabular}{|c|c|c|c|}
\hline Parameter & Description & Value & Reference \\
\hline$\mu_{h}$ & Death rate of humans & $1 / 25000$ & {$[5]$} \\
\hline$\mu_{v}$ & Death rate of vectors & $1 / 4$ & {$[5]$} \\
\hline$r$ & Recovery rate & $1 / 14$ & Assumed \\
\hline$P_{v h}$ & $\begin{array}{l}\text { Transmission probability from vectors to } \\
\text { humans }\end{array}$ & 0.7 & [4] \\
\hline$P_{h v}$ & $\begin{array}{l}\text { Transmission probability from humans to } \\
\text { vectors }\end{array}$ & 0.07 & [4] \\
\hline$b$ & Bites per mosquito per day & 0.3333 & {$[4]$} \\
\hline$C_{v h}$ & Effective contact rate from vectors to humans & 0.2333 & - \\
\hline$C_{h v}$ & Effective contact rate from humans to vectors & 0.0233 & - \\
\hline$P$ & Vaccination coverage & 0.8 & Assumed \\
\hline$v$ & Vaccination rate & 1 & Assumed \\
\hline$\theta$ & Vaccine efficacy & 0.8 & Assumed \\
\hline$n$ & Number of vectors per capita & 10 & Assumed \\
\hline
\end{tabular}




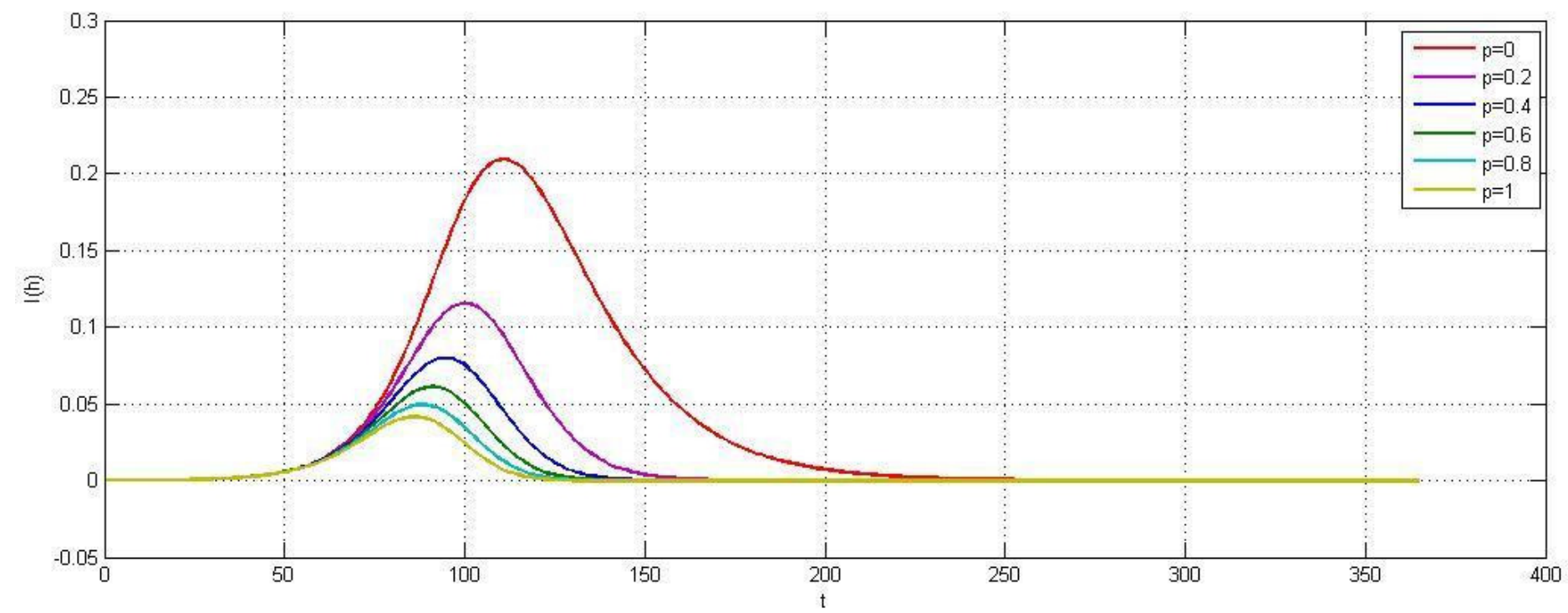

Figure 2: The variation of the infected human profile with respect to the variation of $\mathrm{p}$.

Figures 2 and 3 illustrate the variation of the pools of infected humans $\left(I_{h}\right)$ and infected vectors $\left(I_{v}\right)$ respectively over a period of one year for varying values of $\mathrm{p}=0,0.2,0.4$, $0.6,0.8,1.0$. Further, Figures 4 and 5 present the variation of the reservoirs of infected humans $\left(I_{h}\right)$ and infected vectors $\left(I_{v}\right)$ respectively over a period of one year for varying values of $\theta=0,0.2,0.4,0.6,0.8,1.0$.

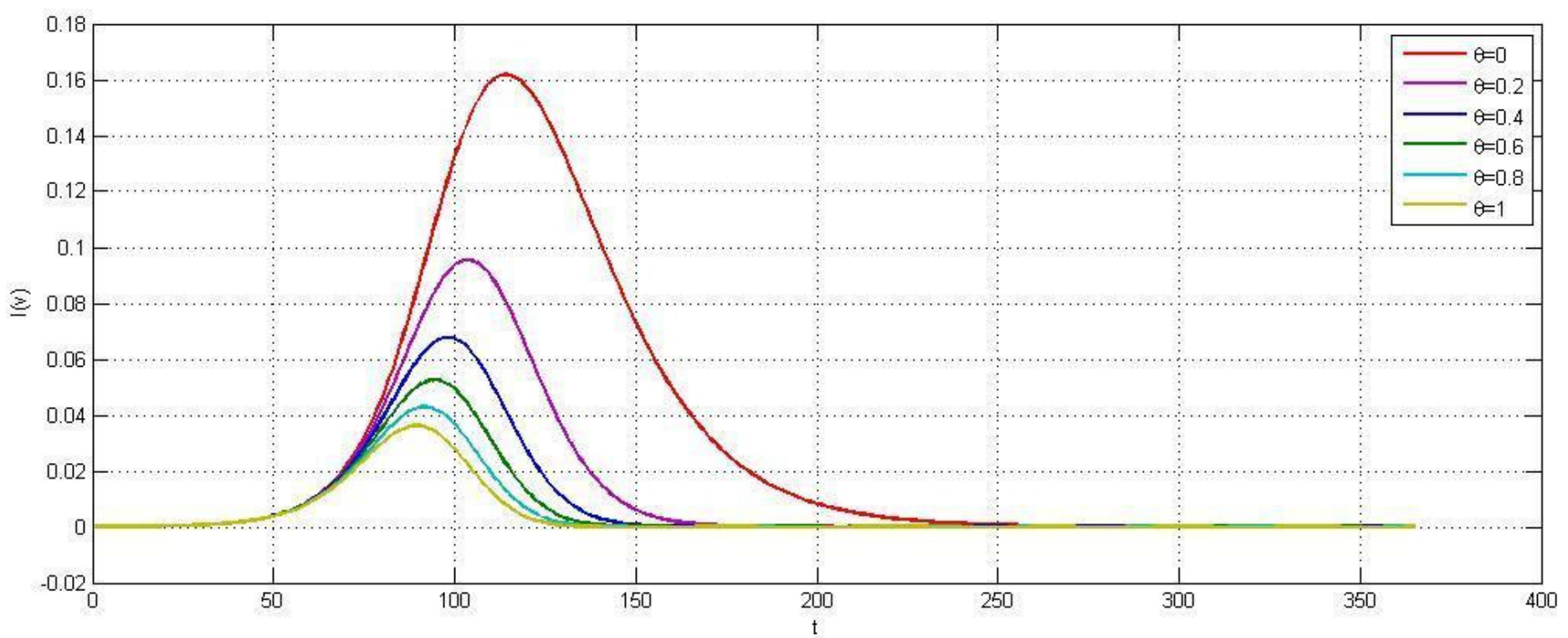

Figure 3: The variation of the infected vector profile with respect to the variation of $p$. 


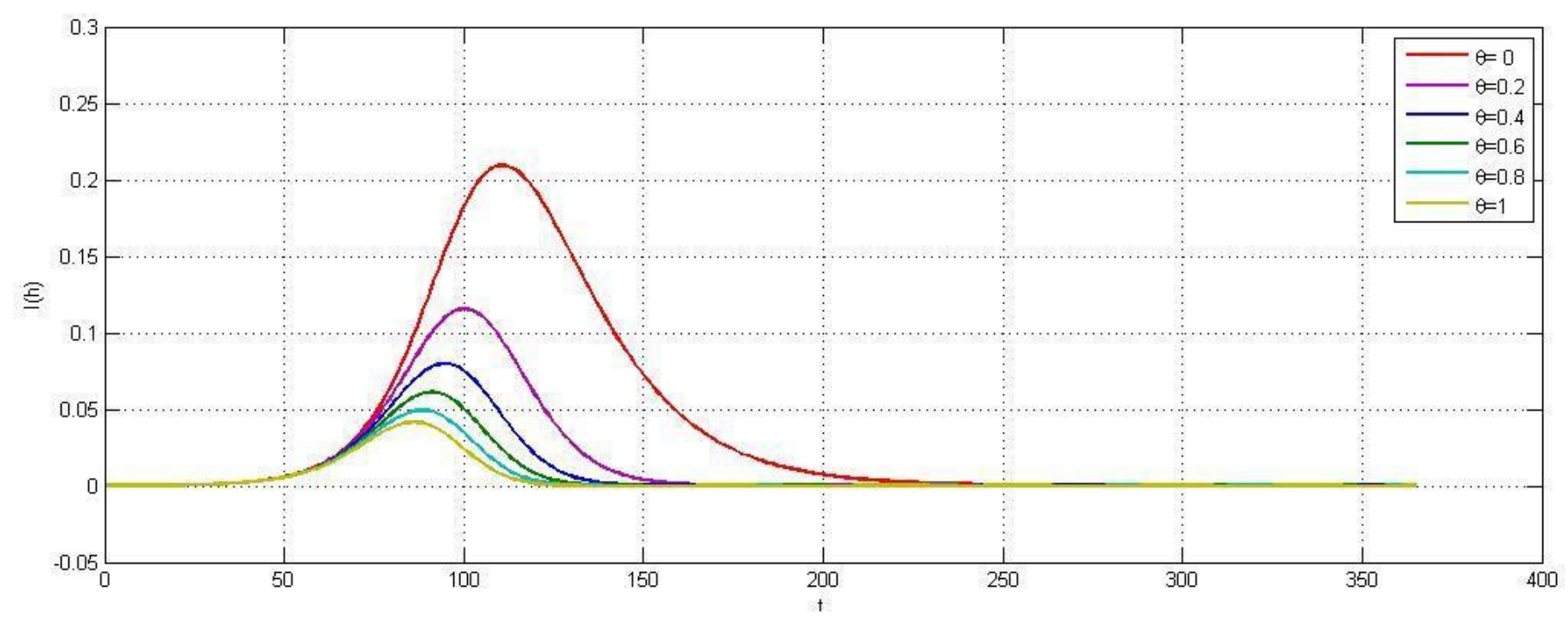

Figure 4: The variation of the infected human profile with respect to the variation of $\theta$.

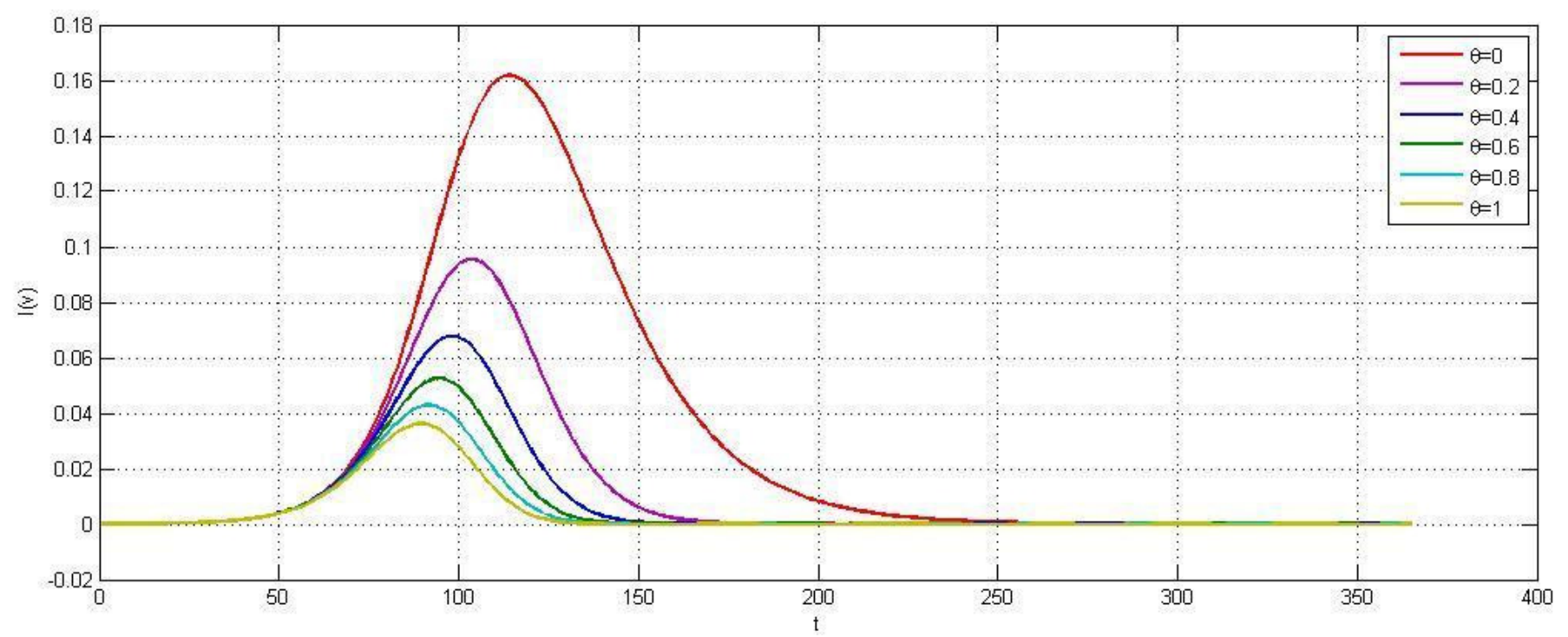

Figure 5: The variation of the infected vector profile with respect to the variation of $\theta$.

Figures 2 and 3 propose that the higher the vaccination coverage (p), the shorter the peak point of the outbreak over the period concerned. Moreover, Figures 4 and 5 signify that the stronger the efficacy of the vaccine, the lower the number of infections in both human and vector populations. So, the Figures 2, 3, 4, and 5 establish the importance of vaccination as an effective measure of disease control. It is also important that the vaccine efficacy has an almost equal impact on the reduction of infections as the vaccination coverage. 


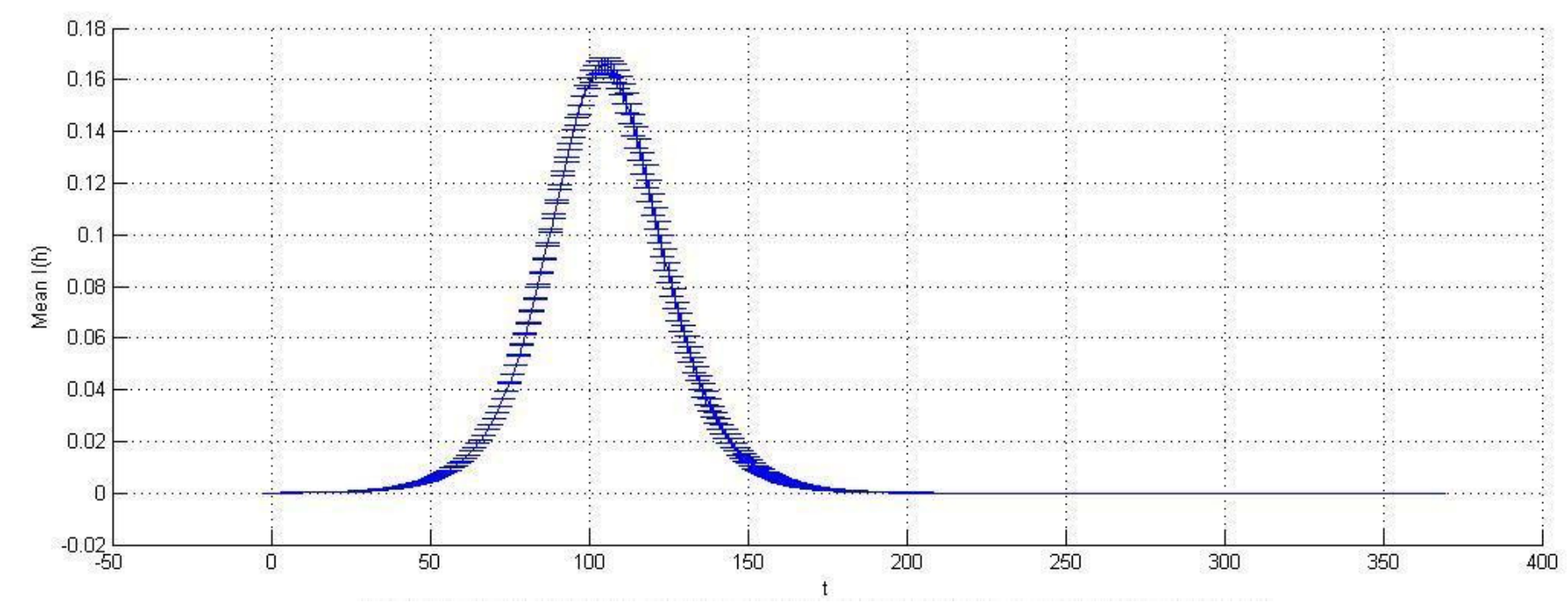

Figure 6: The variation of the infected human profile under the random variation of $p$ over $[0.6,0.8]$.

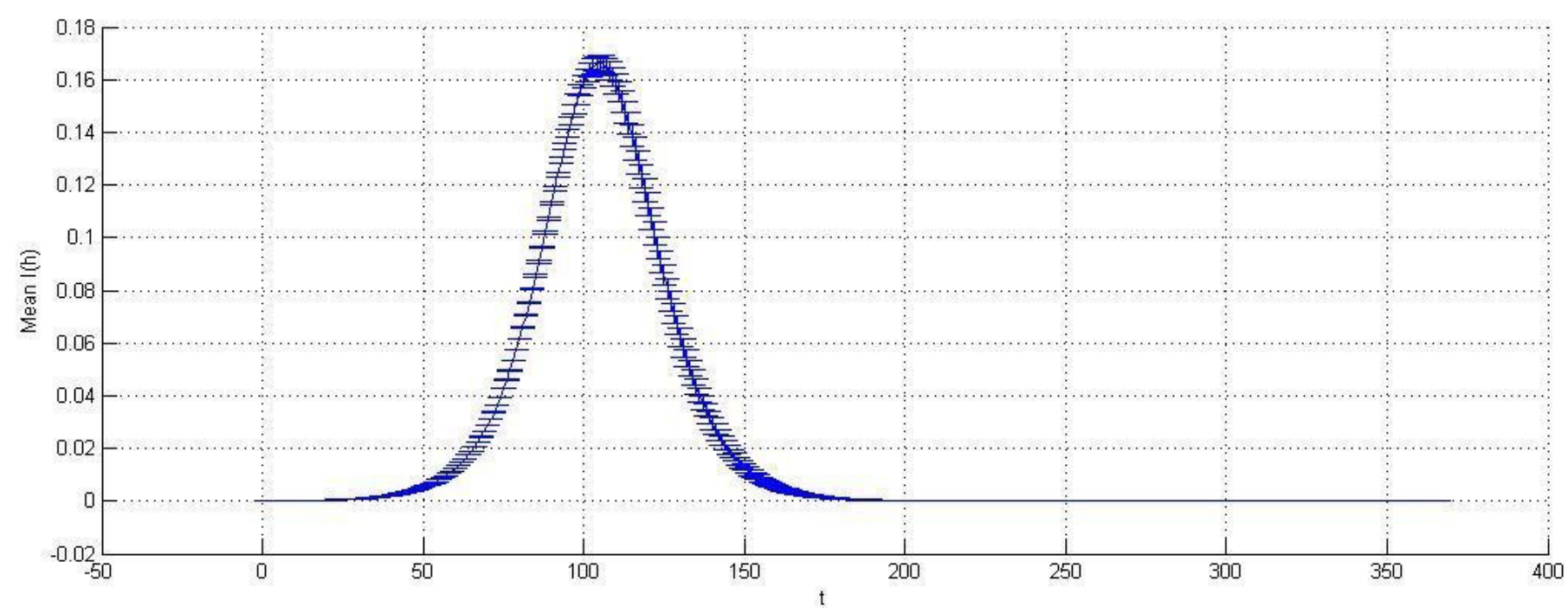

Figure 7: The variation of the infected human profile under the random variation of $\theta$ over $[0.6,0.8]$.

Figures 6 and 7 simulate the variation in the mean value of the size of the infected human population over a period of one year with respect to the random variation of $p$ and $\theta$ respectively over the interval $[0.6,0.8]$. The mean curves with error bars in Figures 6 and 7 reveal that the points on the mean curves are not subject to much variation thus establishing the reliability of the mean values on the curve. In fact, the variations almost disappear after a few days following the peak points. It can further be observed that the shape of the mean curves generated by the random variation of $p$ and 
$\theta$ (Figures 6 and 7 respectively) over $[0.6,0.8]$ are quite the same as those in Figures 2 and 4. This accounts for the reliability of the curves in Figures 2 and 4, and hence it is appropriate to expect such a behaviour in general for potential epidemic situations.

Figures 8 and 9 present the phase plane trajectories of susceptible humans vs. infected humans and infected vectors vs. infected humans respectively over a period of one year for varying values of $\mathrm{p}=0,0.2,0.4,0.6,0.8,1.0$.

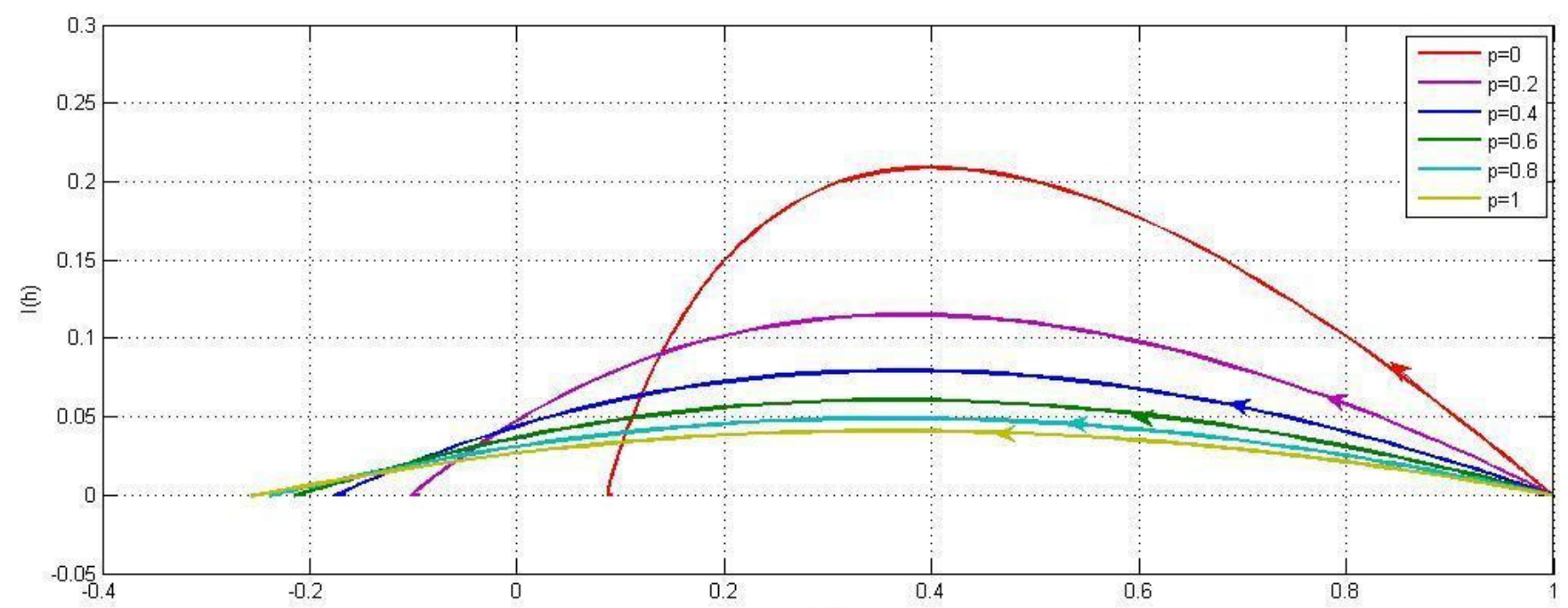

Figure 8: The $S_{h}-I_{h}$ phase plane portrait with respect to the variation of $\mathrm{p}$.

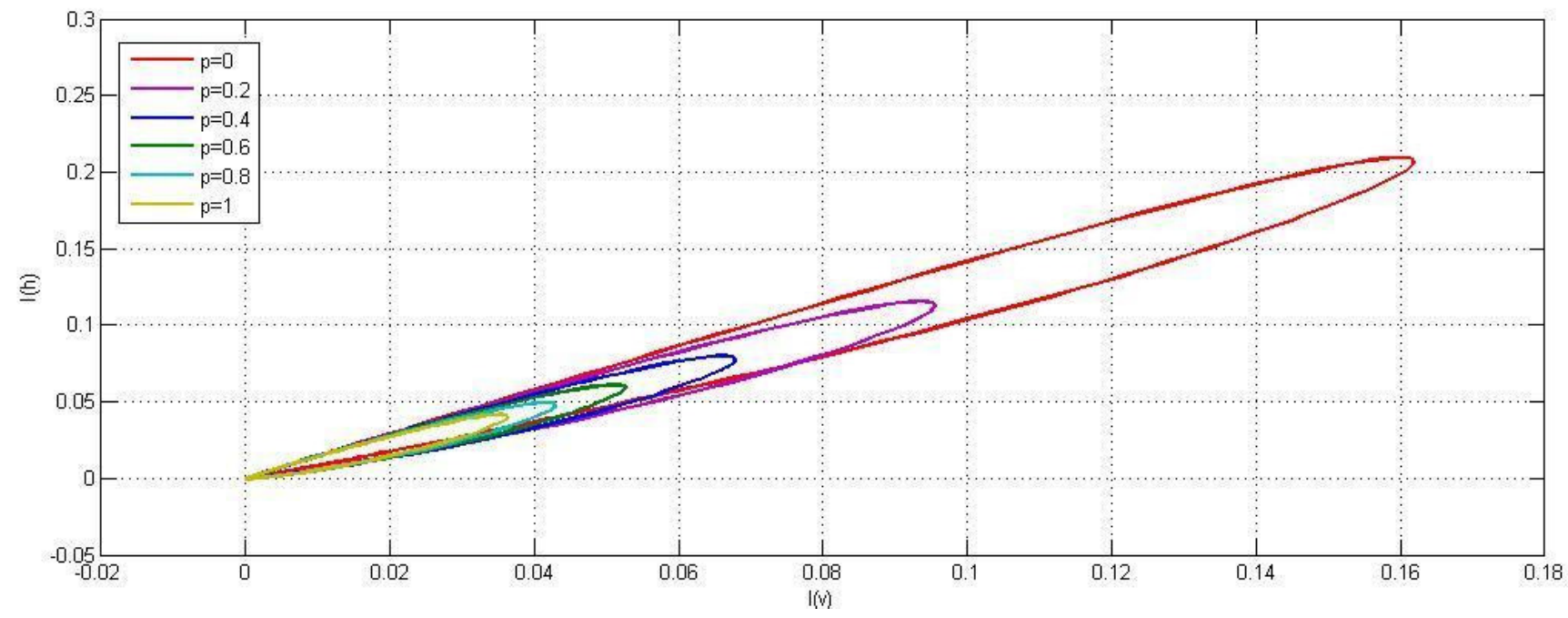

Figure 9: The $I_{v}-I_{h}$ phase plane portrait with respect to the variation of $\mathrm{p}$.

The phase diagrams in Figure 8 read from right to left, because the reservoir of susceptibles is initially at a proportion closer to 1 , and then it starts depleting with time as they become infected with the virus. It can be observed that in Figure $8, I_{h}$ increases 
steadily up to a maximum value as $S_{h}$ decreases, and then $I_{h}$ dwindles a bit more rapidly with $S_{h}$ approaching zero. In the curve corresponding to $\mathrm{p}=0$, we observe that the epidemic ends before all the susceptibles disappear. Further, the curves pertaining to the increasing values of $p$ reveal that the magnitude of the epidemic is comparatively lower for higher values of the vaccination coverage (p).

Figure 9 demonstrates that the number of infected humans increases as the pool of infectious mosquitoes grows. The curves in Figure 9, hence, establish the positive correlation between the variables $I_{h}$ and $I_{v}$. Moreover, the phase diagram in Figure 9 also substantiates the fact that the higher the vaccination coverage (p), the lower the intensity of the epidemic.

\section{CONCLUSION}

In the present paper, we used an SIR type compartmental model in order to analyze the dengue disease dynamics in the single strain structure under the effect of hypothetical vaccination. We were able to derive certain important results concerning the spread and prevalence of dengue epidemics through the mathematical analysis and numerical simulations carried out under the current study. The findings of the paper suggest that potential dengue epidemics can be controlled to a great extent with the administration of a considerably effective vaccine to a large proportion of the population. It is also emphasized that enhancing the efficacy of the vaccines is as important as increasing the vaccination coverage. Further, the importance of vector control measures in addition to the vaccination of hosts is also highlighted in the paper.

Most importantly, the model and analysis used herein may be modified appropriately in order to suit different scenarios. This model, however, is based on the assumption that a person who recovers from a dengue infection, will not be re-infected with the virus (i.e. it is assumed that only one dengue strain is present in the scenario). So, it is possible to further extend the model to a two-subtype structure in order to explain the dynamics of multi-strain co-existence. It is anticipated that this research project will prove to be interesting in the context of the dire need for a productive vaccine in the elimination of dengue disease infections. So, we wish to emphasize the significance of an effective vaccine in the global dengue prevention goals, and also to highlight the strengths of compartmental models in the mathematical modelling of infectious diseases epidemiology. 


\section{REFERENCES}

[1].Aguiar, M., Ballesteros, S., Kooi, B.W., and Stollenwerk, N., (2011). The role of seasonality and import in a minimalistic multi-strain dengue model capturing differences between primary and secondary infections: complex dynamics and its implications for data analyses, Journal of Theoretical Biology, 289:181-196.

[2]. Aguiar, M., Stollenwerk, N. and Haltstead, S., (2016). The Impact of the Newly Licensed Dengue Vaccine in Endemic Countries, PLoS: Neglected Tropical Diseases, 10(12): e0005179.

[3]. Bakach, I., (2015). A survey of mathematical models of Dengue fever. MSc thesis, Georgia Southern University, Statesboro, Georgia, USA.

[4].Daozhou, G., Yijun, L., Daihai, H., Travis, P. C., Yang, K., Gerardo, C., and Shigui, R. (2016). Prevention and Control of Zika as a Mosquito-Borne and Sexually Transmitted Disease: A Mathematical Modeling Analysis, Scientific Reports, 6: 28070.

[5].Derouich, M., Boutayeb, A., and Twizell, E. (2002). A model of dengue fever, BioMedical Engineering Online, 2:4.

[6]. Esteva, L., and Vargas, C. (1998). Analysis of a Dengue Fever Transmission Model', Journal of Bioscience, 150:131-151.

[7]. Nuraini, N., Soewono, E., and Sidarto, K.A. (2007). Mathematical Model of Dengue Disease Transmission with Severe DHF Compartment, Bulletin of the Malaysian Mathematical Sciences Society, 30: 2(143-157).

[8]. Shen, Y. (2014). Mathematical Models of Dengue Fever and Measures to Control It. PhD thesis, College of Arts and Sciences, Florida State University, Tallahassee, Florida, USA.

[9]. Side, S. and Noorani, S. (2013). A SIR Model for Spread of Dengue Fever Disease (Simulation for South Sulawesi, Indonesia and Selangor, Malaysia), World Journal of Modelling and Simulation, 9(2): 96-105. 
[10]. Watmough, J., (2008). Computation of the basic reproduction number. Lecture note for MITACS-PIMS Summer School on Mathematical Modelling of Infectious Disease, University of Alberta, Edmonton, Alberta, Canada.

[11]. Wikipedia, The Free Encyclopedia. (2017). Basic reproduction number. [online] https://en.wikipedia.org/wiki/Basic_reproduction_number (Accessed 8 December 2017).

[12]. Wikipedia, The Free Encyclopedia. (2017). Dengue fever. [online] https://en.wikipedia.org/wiki/Dengue_fever (Accessed 23 September 2017).

[13]. World Health Organization. (2017). Dengue and severe dengue-Fact sheet 117. [online] http://www.who.int/mediacentre/factsheets/fs117/en/ (Accessed 5 November 2017).

[14]. World Health Organization. (2017). Dengue vaccine research. [online] http://www.who.int/immunization/research/development/dengue_vaccines/en/ (Accessed 25 September 2017).

[15]. Yaacob, Y. (2007). Analysis of a dengue disease transmission model without immunity', MATEMATIKA, 23:75-81. 